\title{
Optimal modulation and coding for spatial multiplexing under flat fading channel.
}

\author{
Monika Jain, Student, M.Tech. (ECE) (B.M.I.E.T. Sonipat), Sandeep Singh and Megha \\ Gupta, (B.M.I.E.T. Sonipat)
}

\begin{abstract}
In spatial multiplexing i.e., multiple-input multiple-output (MIMO-OFDM) system, coding schemes can be employed in link adaptation algorithms to improve data rates, effective use of allocated frequency spectrum and further to mitigate the effect of dynamic nature of the channel are used. Coding schemes can be used in link adaptation algorithms [1]. These loading algorithms adapt the system parameters according to the channel state information (CSI). It also improves the bit error rate (BER) performance and minimizes the total power input at the transmitter while maintaining a defined data-rate constraint. Ultimately, these algorithms gives better performance in the flat fading channel which will also be confirmed by simulation results.
\end{abstract}

Keywords:- BER, Gray code, MISO, M-PSK, SISO, SNR, TCM

\section{INTRODUCTION}

The permeating use of wireless communication is fueling the need for higher data rates and channel capacity. OFDM technology is capable of solving the difficult inter-symbol interference problem encountered with high data rates across multiple channels. The multi-carrier technology in form of MIMO-OFDM is widely organized as one of the most promising access scheme for the wireless network over SISO-OFDM without wasting spectrum source. By dividing the bandwidth into the small frequency bands, the data can be transmitted across multiple narrowband channels but it suffer only from flat fading [6]. The orthogonality of the sub-carriers in OFDM makes it possible to use different modulation schemes for different subcarriers.

A way to combat with flat fading is to adapt number of bits and power level on each sub-carrier according to CSI [2]. Here if, using adaptive modulation and MIMO-OFDM with appropriate coding schemes to create an effective algorithm can result in efficient BER performance for broadband wireless MIMO-OFDM system. The objective of using link adaptation scheme is to get best possible system performance based on CSI. The paper is organized as follows:-Section 2 introduce system model, section 3 is about adaptive modulation and Section 4 introduce link adaptation criterion. Finally, the simulation results and a conclusion is presented in section 5 and 6.

\section{SYSTEM MODEL}

In a MIMO-OFDM system with $\mathrm{N}$ subcarriers (or tones) the individual data streams are first passed through OFDM modulators which perform an IFFT on blocks of length $\mathrm{N}$ followed by a parallel-to-serial conversion. A cyclic prefix (CP) containing a copy of the last samples of the parallel-to-serial converted output of the N-point IFFT is then prepended. The resulting OFDM symbols are launched simultaneously from the individual transmit antennas. The $\mathrm{CP}$ is essentially a guard interval which serves to eliminate interference between OFDM symbols and turns linear convolution into circular convolution such that the channel is diagonalized by the FFT. In the receiver the individual signals are passed through OFDM demodulators which first discard the CP and then perform an N-point FFT. The outputs of the OFDM demodulators are finally separated and decoded. The assumption of the length of the CP being greater or equal than the length of the discrete-time base-band channel impulse response guarantees that the MIMO fading channel indeed decouples into a set of parallel MIMO fading channels [5].

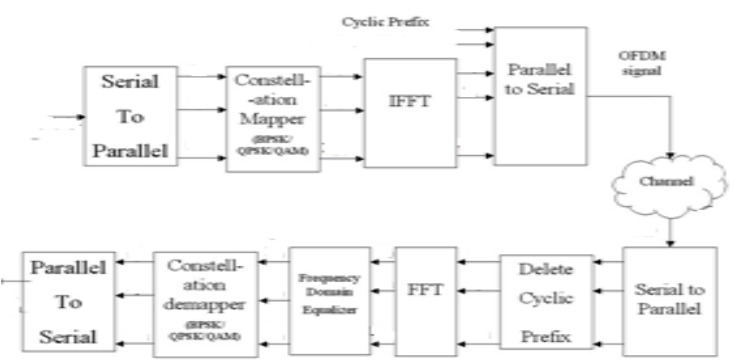

Fig. 1 System Model block diagram 


\section{ADAPTIVE MODULATION}

Adaptive modulation in OFDM is a technique of using different modulation modes in different subcarriers depending on the instantaneous signal to noise (SNR) ratio for each sub-channel. Channel conditions are estimated at the receiver and CSI is sent to the transmitter to adapt the transmission accordingly. Parameters like transmitted power required, numbers of bits on each sub-carrier modulation scheme or combination of these can be adapted according to the channel condition. Nearly, all communication system requires some target BER not to be exceeded. It is possible to assume an average BER or an instantaneous BER as a constraint. Another assumption that can be made is the knowledge of CSI for each sub-carrier. When no adaption is done, the system present low SNR. To maintain the targeted BER, it is necessary to compensate the state of channel by adapting the transmitted power level.

The nature of OFDM allows the signal to modulate in amplitude and phase. In this paper we will obtain our result from phase shift keying modulation (M-PSK), where the amplitude of the transmitted signal is constrained to remain constant, thereby yielding a circular constellation. By allowing the amplitude to vary with phase another modulation scheme called quadrature amplitude modulation (QAM) can also be obtained.

\section{LINK ADAPTATION CRITERION}

Loading algorithms in multicarrier systems computes number of bits ${ }^{\circ} b_{n}$, and power ' $\varepsilon_{n}$, required for transmitting these allocated bits on each sub-channel. There are two types of loading algorithms i.e., those which try to maximize data rate while maintaining a target BER constraint and others which try to maximize performance at a given fixed data rate.

In this paper, two algorithms are used for adaptive bit and power loading i.e., Chow's algorithm and Compello's algorithm. Initially each sub-channel was allocated energy using Chow's algorithm and at the same time it was allocated maximum number of bits allowable per sub-channel. Then bit allocation was done using Compello's algorithm.

IV.1. Chow's Algorithm:- Chow was able to verify that an "on/off" energy distribution, as long as it used the same or nearly the same transmission band as water-filling, exhibits negligible loss with respect to the exact water-filling shape[4].The procedure is summarized as follows:

a).Compute the sub-channel signal to noise ratios based on the formula

$S N R(i)=(\text { Subcarriers gain })^{2} /($ noise $\times$ gap $)$ Where $S N R(i)$ is the signal to noise ratio for ratio for each subchannel.

b).Compute the number of bits for the $i^{\text {th }}$ sub-channel based on the formula:

$$
\hat{b}(i)=\log _{2}(1+S N R(i) / \Gamma)
$$

Here $\hat{b}(i)$ is the number of allocated on each subcarriers

c).Round the value $\hat{b}(i)$ down to integer $b(i)$

d). Restrict $b(i)$ to take values $0,1,2,4,6$ or 8 .

e).Compute the energy for the $i^{\text {th }}$ subcarrier based on the number of bits initially assigned to it using the formula:

$$
\begin{aligned}
& \qquad e_{i}(b(i))=\left(2^{b(i)}-1\right) / G N R(i) \\
& \text { Where } G N R(i) \text { is } S N R(i) / g a p
\end{aligned}
$$

The 'gap' works as tuning parameter, different values of 'gap' gives different ' $E_{b} / N_{0}$ ' ratio for given desired number of bits to be transmitted.

IV.2. Compello Algorithm: - The Compello's algorithm is nothing but a one bit B-tightness algorithm [3]. Given the initial bit allocation, the B-tightness simply states the correct number of bits is being transmitted and optimized the bit allocation.

A $\beta$-tightening $\left(\beta_{\mathrm{T}}\right)$ algorithm is: 

a).Set
$\hat{b}=\sum_{n=1}^{N} b_{n}$
b). While $\hat{b} \neq b$
If $\hat{b}>b$
(1)
$n \leftarrow \arg \left\{\max _{1 \leq n \leq N}\left[e_{n}\left(b_{n}+\beta\right]\right\}\right.$
(2) $\hat{b} \leftarrow \hat{b}-\beta$
(3)
$b_{n} \leftarrow b_{n}-\beta$
Else
(1)
$m \leftarrow \arg \left\{\min _{1 \leq n \leq N}\left[e_{n}\left(b_{n}+\beta\right]\right\}\right.$
(2) $\hat{b} \leftarrow \hat{b}+\beta$
(3) $b_{n}=b_{n}+\beta$

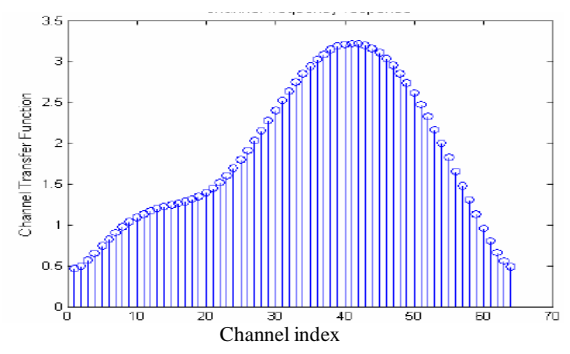

(a) Channel Frequency Response

For instance, these two algorithms can be configured in following figures. This figure 2 illustrates the typical channel frequency response, the discrete bit allocation to each tone, and the corresponding energy on each tone.

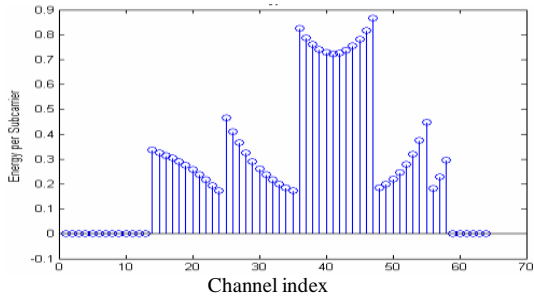

(b)Discrete energy allocation

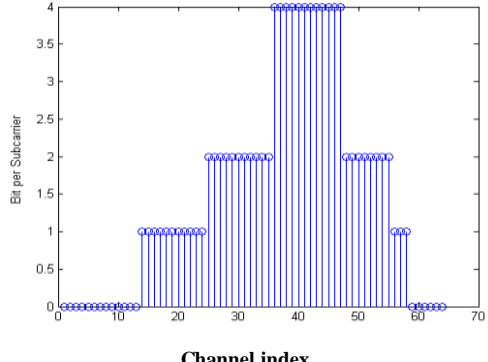

(c)Discrete allocation bit

Fig. 2. Optimal bit and power allocation (a) Channel frequency response (b) Energy Allocation (c) Discrete Bit Allocation

\section{SIMULATION AND RESULTS}

In this section simulation result for adaptive M-PSK, adaptive M-PSK with gray coding and adaptive M-PSK with TCM under flat fading Rayleigh channel is presented. Functioning of the channel without any 
delay is necessary and assumed during the simulation at the transmitter and the receiver for the implementation of the link adaptation algorithms. The entire system is considered as discrete time system.

Figure 3 and 4 shows the graphical representation of BER vs Eb/No performance under flat fading channel (Rayleigh channel) for SISO-OFDM and MIMO-OFDM(2X2 antenna).

The system with 64 subcarriers, 64 OFDM symbol time periods, and 16 symbol periods for guard time. The parameters were held constant throughout the simulation. M-PSK adaptive modulation for each subcarrier is used whereas 2 PSK, 4PSK, 16PSK, 64PSK AND 256PSK the usable modulation modes configurations.

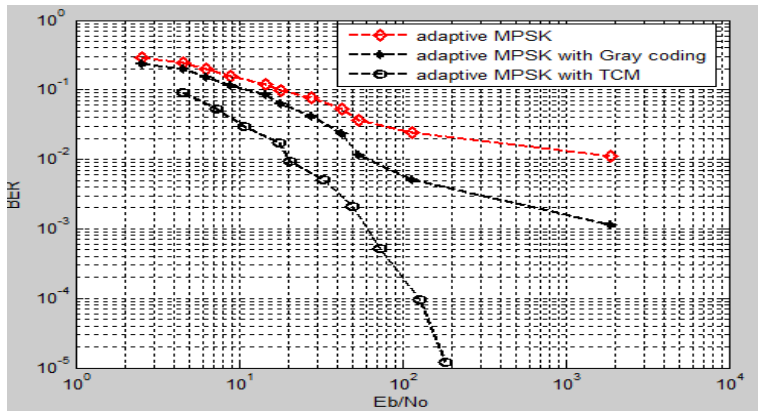

Fig.3. BER vs Eb/No values for adaptive MPSK, adaptive MPSK with Gray coding and adaptive MPSK with TCM SISO-OFDM system under flat fading channel.

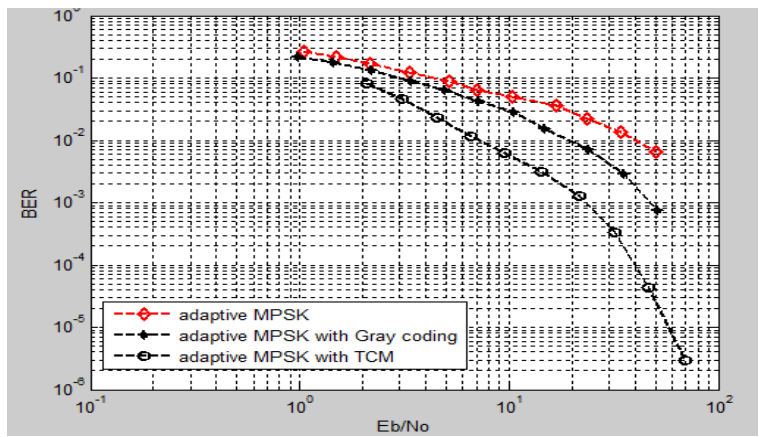

Fig.4. BER vs Eb/No graph for Adaptive MPSK . adaptive MPSK with gray coding and adaptive MPSK with TCM for MIMO-OFDM system under flat fading channel.

\section{CONCLUSION}

Link adaptation algorithms with TCM and Gray code results in improvement of BER performance for MIMO-OFDM system over SISO-OFDM system with M-PSK. The simulation result indicates that coded adaptive OFDM systems are better as compared to non coded adaptive OFDM systems.

\section{REFERENCES}

[1]. Guoting Lai Junxun Yin Fan Lin Huawen Yu, "Performance of Adaptive Bit and Power Allocation MIMO-OFDM System Based on Greedy Algorithm”, IEEE Wireless Communication and Networking Conference, vol. 1 , 2005 pp, 44-49.

[2]. Liu Jiancai Yang Wanwan Zheng Weili Wang Yafu Ye Zhihui Xu Jiakai and Ning Xinbao "Adaptive bit and power loading algorithm with low complexity in MIMO-OFDM systems", IEEE international conference, 2009,

[3]. J. Campello: "Optimal discrete bit loading for multicarrier modulation systems", Proc. of the IEEE Int. Symp. On Information Theory, p. 193, Cambrige, MA, USA, Aug. 1998.

[4]. Yu Qing and Wang Yi “ Improved Chow Algorithm Used in Adaptive OFDM system” DOI 10.1109/ CMC .2010.212@2010 IEEE.

[5]. H. B $\square$ olcskei and A. J. Paulraj, "Space-frequency coded broadband OFDM systems," in Proc.IEEE WCNC-2000, Chicago, IL, Sept. 2000, pp. 1-6.

[6]. Hung Tuan Ngugen, Jogen Bach Andersen and Gert Frolund Prdersen, " On the Performance of Link Adaptation Techniques in MIMO systems, Wireless Personal Communications, Springer Publications, 2006 